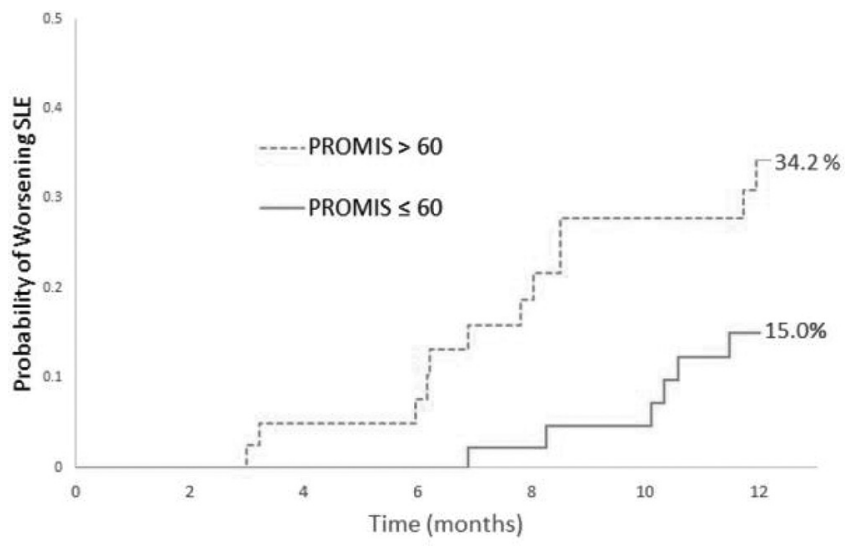

Abstract 55 Figure 1 Poor sleep quality at prior visit predicts SLE flare activity

previous visit predicted worsening SLE activity at the next visit (figure 1). Over a 12 month period, the probability of SLE activity worsening was $21.4 \%$ overall, $34.2 \%$ for SRI $>60$, and $15.0 \%$ for SRI $\leq 60(p=0.024)$.

Conclusions Our study reinforces that patients with SLE report worse subjective sleep compared to the general population, and patients with active SLE have worse sleep than patients with inactive SLE. Our longitudinal data demonstrate that poor sleep predicts worsening SLE disease activity. Thus, variation in subjective sleep may have an important role in SLE flares.

Funding Source(s): AMH and PC: Washington University School of Medicine Mentors in Medicine Program, Rheumatology Research Foundation Resident Research Preceptorship Award. YSJ: NIH/NINDS K23NS089922, NIH/NIA R34AG056639, UL1RR024992, and KL2-TR000450. AHJK: NIH/NIAMS R21AR069833, Midwest Strategic Pharma-Academic Research Consortium, and the Doris Duke Foundation Fund for Retaining Clinical Scientists Program.

\section{SELF-REPORTED INDIRECT COSTS ARE UNDERESTIMATED IN A CANADIAN COHORT OF PATIENTS WITH SLE}

${ }^{1}$ Ann E Clarke*, ${ }^{1}$ Megan RW Barber, ${ }^{2}$ Yvan St Pierre, ${ }^{3}$ Murray B Urowitz, ${ }^{3}$ Dafna D Gladman, ${ }^{2}$ Sasha Bernatsky, ${ }^{2}$ Evelyne Vinet, ${ }^{2}$ Christian Pineau, ${ }^{4} J o h n$ Hanly, ${ }^{5}$ Christine Peschken, ${ }^{6}$ Paul Fortin, ${ }^{1}$ Michelle Jung, ${ }^{1}$ Claire Barber, ${ }^{7}$ Susan Elliott. ${ }^{1}$ Division of Rheumatology, Cumming School of Medicine, University of Calgary; ${ }^{2}$ Department of Medicine, Division of Rheumatology, Faculty of Medicine, McGill University; ${ }^{3}$ Krembil Research Institute, University of Toronto; ${ }^{4}$ Division of Rheumatology, Department of Medicine, Dalhousie University; ${ }^{5}$ Faculty of Medicine, Department of Internal Medicine, University of Manitoba; ${ }^{6}$ Centre de Recherche, Université Laval; ${ }^{7}$ Department of Geography and Environmental Management, University of Waterloo

\subsection{6/lupus-2019-Ism.56}

Background Indirect costs (IDC) of SLE reflect lost productivity in work force (WF) and non-WF activities and can be expressed as: 1) patient self-report of lost productivity or 2) the difference between productivity of an age-and-sex matched general population and the patients stated productivity. We assess IDC calculated by both methods in a Canadian-wide cohort and compare IDC, stratified by damage, across methods. Methods Patients fulfilling the ACR or SLICC Classification Criteria from 6 centres were enrolled. Participants completed a validated questionnaire on lost productivity. Lost
Abstract 56 Table 1 Indirect cost calculations by Method \#1 (difference between patient self-report of expected versus actual productivity) and Method \#2 (difference between time worked by matched general population versus actual patient productivity)

\begin{tabular}{llll}
\hline SDI & $\begin{array}{l}\text { IDC Method \#1 } \\
\text { Mean }(95 \% \mathrm{Cl})\end{array}$ & $\begin{array}{l}\text { IDC Method \#2 } \\
\text { Mean }(95 \% \mathrm{Cl})\end{array}$ & $\begin{array}{l}\text { Difference between } \\
\text { Method \#1 and \#2 } \\
\text { Mean }(95 \% \mathrm{Cl})\end{array}$ \\
\hline $\mathbf{0}$ & $\$ 17109$ & $\$ 19326$ & $\$ 2217^{1}$ \\
1 & $(\$ 13,021, \$ 21,197)$ & $(\$ 14,936, \$ 23,717)$ & $(\$-3782, \$ 8216)$ \\
& $\$ 19937$ & $\$ 25963$ & $\$ 6027^{2}$ \\
2 & $(\$ 16,158, \$ 23,715)$ & $(\$ 21,764, \$ 30,163)$ & $(\$ 378, \$ 11,676)$ \\
& $\$ 22825$ & $\$ 31733$ & $\$ 8908$ \\
3 & $(\$ 18,479, \$ 27,171)$ & $(\$ 27,401, \$ 36,064)$ & $(\$ 2772, \$ 15,044)$ \\
4 & $(\$ 19398$ & $\$ 30022$ & $\$ 10624$ \\
& $\$ 26159$ & $(\$ 23,682, \$ 36,362)$ & $(\$ 2293, \$ 18,956)$ \\
$\geq 5$ & $(\$ 19,195, \$ 33,124)$ & $(\$ 29,819, \$ 45,605)$ & $(\$ 1027, \$ 22,079)$ \\
& $\$ 25265$ & $\$ 41220$ & $\$ 15,955,2$ \\
& $(\$ 19,647, \$ 30,883)$ & $(\$ 35,456, \$ 46,984)$ & $(\$ 7906, \$ 24,004)$ \\
\hline
\end{tabular}

${ }^{1}$ The difference between the between method difference at $\mathrm{SDI} \geq 5$ and $\mathrm{SDI}=0$ is $\$ 13738$ $(95 \% \mathrm{Cl}, \$ 3700, \$ 23,777)$.

${ }^{2}$ The difference between the between method difference at $\mathrm{SDI} \geq 5$ and $\mathrm{SDI}=1$ is $\$ 9928$ $(95 \% \mathrm{Cl}, \$ 95, \$ 19,762)$.

productivity was calculated as: 1) the difference between the time patients reported they expected they would engage in WF and non-WF activities if not ill versus the time they reported working and 2) the difference between the time worked by an age-and-sex matched general population in WF and non-WF activities versus the time patients reported working. IDC were valued using age-and-sex-specific wages from the Statistics Canada General Social Survey. IDC from nonWF activities were valued using opportunity costs (i.e., expected WF earnings, rather than expected earning of service workers). Annual IDC (2017 Canadian dollars) associated with damage measured on the SLICC/ACR Damage Index (SDI) were obtained from multiple regressions adjusting for age, race/ethnicity, and disease duration.

Results 1368 patients participated, 90.4\% female, 70.9\% Caucasian, mean age at diagnosis 33.0 years (SD 13.5), mean SLE duration 16.8 years (SD 11.6), mean SLE Disease Activity Index (SLEDAI-2K) 2.15 (SD 3.07), and mean SDI 1.54 (SD 1.87). IDC by method \#1 versus \#2, stratified by SDI, are shown in table 1 . Although at $\mathrm{SDI}=0$, mean predicted IDC did not differ between methods, for $\mathrm{SDI}=1$ through SDI 5, IDC by method \#2 were greater.

Conclusions IDC by method \#2 were greater for SDIs 1 through 5 and the difference between methods increased significantly between lower and higher SDIs ( $<2$ versus 5). Our results suggest that IDC calculated by comparing the patients actual productivity to their self-report of expected productivity versus the productivity of an age-and-sex-matched general population leads to underestimation, which is not associated with damage. Patients expectations of productivity appear to plateau with increasing damage and do not reflect their likely productivity if they were not ill. Hence, IDC should not only rely on patients self-report of lost productivity, but should also incorporate a comparison of the patients productivity with the actual productivity of a matched general population. Funding Source(s): Canadian Initiative for Outcomes in Rheumatology cAre (CIORA) 